\title{
On the Dependence of Spectral Turnover on Linear Size of Compact Steep Spectrum Radio Sources
}

\section{Ezeugo Jeremiah Chukwuemerie}

Department of Physics and Industrial Physics, Nnamdi Azikiwe University, Awka, Nigeria

Email address:

chuksemerie@yahoo.com

To cite this article:

Ezeugo Jeremiah Chukwuemerie. On the Dependence of Spectral Turnover on Linear Size of Compact Steep Spectrum Radio Sources. International Journal of Astrophysics and Space Science. Vol. 3, No. 2, 2015, pp. 20-25. doi: 10.11648/j.ijass.20150302.12

\begin{abstract}
Frequency peak/linear size $\left(v_{p}-D\right)$ relation gives the strongest correlation among all the currently observable physical properties of Compact Steep Spectrum Sources (CSSs). This strong correlation suggests that the spectral turnover constitutes a characteristic signature of dense gases around the CSS sources which may be used to constrain physical mechanisms that govern the dynamical evolution of CSS sources. We have therefore, carried out statistical analyses to ascertain whether the observed $v_{p}-D$ correlation is real or an artifact arising from a possible spectral turnover/radio luminosity $\left(v_{p}-P\right)$ and/or linear size/radio luminosity $(D-P)$ correlations. Our results show only a marginally significant $v_{p}-P$ correlation (correlation coefficient, $r \approx 0.3$ ) and apparently little or no $D-P$ correlation. This suggests that the mechanism for the spectral turnover lies mainly in the source size according to the relation, $v_{p} \sim D^{-0.7}$, with correlation coefficient, $r \approx-0.9$.
\end{abstract}

Keywords: Galaxies, Quasars, Radio, Luminosity, Redshift

\section{Introduction}

Compact steep spectrum sources are made up of small scaled radio galaxies and radio-loud quasars with projected linear sizes which range from less than $1 \mathrm{kpc}$ to $20 \mathrm{kpc}$ assuming Hubble's constant, $H_{0}=75 \mathrm{Kms}^{-1} \mathrm{Mpc}^{-1}$, and deceleration parameter, $q=0$. Their radio luminosity at $5 \mathrm{GHz}$ is greater than $10^{25.5} \mathrm{WHz}^{-1}[1,2]$. They show double, triple and core-jet morphologies on the radio maps [3]. They have steep high frequency spectrum of spectral index, $\alpha \geq 0.5,\left(S_{v} \propto v^{-\alpha}\right) \quad[3$ and references therein]. Their frequency spectral turnover is believed to be due to synchrotron self absorption in a compact component with a large magnetic field $[2,4,5,6]$. Generally, they have very low polarization at both radio and optical bands, usually less than one percent $[7,8,9]$. Their proportion is high; about $15-30 \%$, depending on the selection frequency, among distant $(z>0.2)$ radio sources of high luminosity [7,9].

Based on their spectral turnovers, three subclasses of CSS sources exist. They are Low Frequency Peakers (LFPs), which peak at $<0.5 \mathrm{GHz}$; GigaHertz-Peaked Spectrum sources (GPSs), which have the following range of spectral peaks, $0.5 \leq v_{p} \leq 5 \mathrm{GHz}$; and High Frequency Peakers (HFPs) which peak at $>0.5 \mathrm{GHz}$. Moreover, according to their linear sizes, we have the following subclasses: Compact Symmetric
Objects (CSOs) whose linear sizes, D, are $D<1 \mathrm{kpc}$; Medium-sized Symmetric Object (MSOs) whose range of linear sizes is $1 \leq D \leq 15 \mathrm{kpc}$; and Large Symmetrical Objects (LSOs) whose range of linear sizes is $15<D \leq$ $20 \mathrm{kpc}[6,7,10,11,12,13,14,15]$. CSOs, MSOs, and LSOs can be referred to as symmetric CSS sources and present two-sided ejection and are intrinsically very luminous since there is no indication of relativistic beaming [1].

However, some observations have shown that some CSS sources are asymmetric core-jet sources, and can be referred to as asymmetric CSS sources. These asymmetric sources show distorted/complex structures on radio maps; while the symmetric sources exhibit double-lobed structures and have components with similar spectral shape and flux density $[16,17]$. In contrast to what happens to asymmetric sources, the symmetric structure indicates that they are not Doppler-boosted. Even so, they have luminosities comparable to the luminous, edge-brightened, extended double sources.

CSS sources have been suggested to be young, scaled down versions of the classical double sources of Fanaroff-Riley class II type [4,5,6,12,16,18,19]. Moreover, Fanti et al., (1995) have studied a sample of double-lobed CSS sources with linear sizes of a few kpc, which in analogy with the name CSO, named MSOs (Medium-sized Symmetric Objects). They concluded that these are young objects and are the precursors 
of the extended double radio sources. It has been shown that CSS sources with galaxy identifications show properties consistent with the "youth scenario" $[18,20]$. With ages less than $10^{4} \mathrm{yr}$ and having a sub-galactic extent of $<1 \mathrm{kpc}$, the compact doubles (or CSOs as we now call them) are very young radio sources that evolve into extended FR II radio sources on time scales of $10^{6}-10^{8} \mathrm{yr}[4,5,6,10,18,21,22]$. This model suggests that there exists an evolutionary sequence from compact doubles to the more extended doubles; or more generally, from the CSOs through the MSOs and LSOs to the more extended doubles. These three classes all have common properties; they are high luminosity, symmetric structure and are manifestations of the same type of objects seen at different epochs of their evolution $[6,10,18]$.

However, there are indications that at least a fraction of CSS sources may not be as young as initially thought. For instance, extended emissions have been detected in some CSS sources and other observations showed that about $20 \%$ of GPS sources present a diffuse extended radio emission though more resent results indicate that this number could be lower, in the range 5 $-10 \%[9,23]$. In these sources the nuclear activity has been proposed to be a recurrent phenomenon, the diffuse emission being the relic of a previous activity while the compact GPS sources would be the product of a renewed of the host galaxy. Another possibility is that the galaxy has suffered a resent and sudden increase in the gas density near the centre due to an interaction or merger with a gas rich companion. The radio source has been smothered by the dense and clumpy gas that keeps the source confined to the nuclear region $[9,23]$.

Furthermore, images of some GPS sources show distorted morphologies and the presence of dust, suggesting that mergers and galaxy-galaxy interaction played an important role in these objects $[2,3,24,25]$. This corroborates the hypothesis that some CSS sources are confined in the dense nuclear region. In that case CSS sources would not be young objects but have ages comparable to the extended doubles this is referred to as 'Frustration Scenario' [7,12]. Moreover, Kawakatu and Kino, 2007 using analytical model described the evolution of the velocity, pressure, density, and radius of the hot spot as function of slope index of ambient density and the growth rate of head parts of the cocoon.

Among the currently observable physical parameters of CSS sources, a strong correlation exists in the spectral turnover (frequency peak)/linear size relation; and this may contain reasonable information concerning the linear size evolution of the CSS sources [9]. Moreover, a slight correlation is found in the frequency peak/radio luminosity relation. Hence, it behoves one to find which of the two correlations is a primary and which is a secondary effect; and this is the aim of this paper.

\section{Dependence of Linear Size on Radio Luminosity and Redshift}

In any friedmann-Robertson Walker world model, the angular size $(\theta)$ subtended by a source of projected linear size $(D)$ is at a redshift, $z$, given by

$$
\theta=\frac{D(1+z)^{2}}{d_{L}}
$$

Where $d_{L}$ is the luminosity distance which depends on the Hubble's constant, $H_{0}$, and the density parameter, $\Omega_{0}$, according to the relation,

$$
d_{L}=\frac{4 c}{H_{0} \Omega_{0}^{2}}\left\{\Omega_{0} Z+\left(\Omega_{0}-2\right)\left[\sqrt{\Omega_{0} Z+1}-1\right]\right\}
$$

Also, the spectral luminosity, $P$, is related to its spectral flux density, $S_{v}$, at observing frequency, $v$, by

$$
P=S_{v} d_{L}^{2}(1+z)^{\alpha-1}
$$

where $\alpha$ is the spectral index defined by $S_{v} \sim v^{-\alpha}$.

In flux density limited samples, $P$ and $Z$ are tightly correlated due to Malmquist bias and is usually represented in terms of a power-law function [26],

$$
P \sim(1+z)^{\beta}
$$

Analyses of $\theta-z$ relation (1), especially for extended extragalactic sources show that a linear size evolution of the form $D \sim(1+z)^{-x}$ or equivalently, a luminosity dependent linear size evolution of the sort $D \sim P^{q}(1+z)^{-n}$ would be required to interpret the observed $\theta-z$ data $[27,28,29]$. Whether this applies to CSS sources is not clear [27].

It therefore follows, that if there is a $D-P$ or $D-Z$ correlation for the CSS data, then the observed $v_{p}-D$ correlation may simply be an artifact. We investigate this in the next section.

\section{Data Analysis and Results}

The list of 65 samples of CSS sources (31 quasars and 34 galaxies) used in this work, were obtained from [9].

Considering the $v_{p}-D$ plot in figure 1 , it is worthwhile to note that the projected linear size, D, and the frequency peak, $v_{p}$, have the strongest correlation among all the observable parameters of CSS sources and are related according to power-law of the form [9]:

$$
v_{p} \sim D^{-\beta}
$$

with $\beta=-0.67$ and correlation coefficient, $r \approx-0.9$. Hence any good model for the dynamical evolution of radio sources should be able to show a power-law relationship between the linear size and the frequency peak.

Moreover, a marginally significant correlation exists between the frequency peak and radio luminosity $(r \approx 0.3)$; this is shown in figure 2. Furthermore, figure 4 shows $v_{p}-z$ relation with correlation coefficient, $r=0.16$; while figure 5 is $D-z$ plot with correlation coefficient, $r=-0.11$.

If we assume for $v_{p}-P$ that $r \approx 0.3$ is somehow significant enough for a good correlation coefficient, therefore, we ascertain whether the correlation in $v_{p}-D$ relation is primary or a secondary effect. To remove the effect of luminosity, $P$, from the $v_{p}-D$ relation, we apply 
Spearman-rank statistics:

$$
r_{12,3}=\frac{r_{12}-r_{13} r_{23}}{\left[\left(1-r_{13}^{2}\right)\left(1-r_{23}^{2}\right)\right]^{\frac{1}{2}}}
$$

where $r_{12,3}$ is the Spearman-rank correlation coefficient. It tests whether there is a significant correlation between the two observable parameters, 1 and 2 that does not arise from being separately correlated with a third parameter, 3 . This implies that it is used to test for a possible correlation between the first two parameters when the third parameter is kept constant. The statistics varies from -1 (i.e. for a perfect inverse correlation) to +1 (i.e. for a perfect positive correlation) with 0 depicting null correlation. $r_{12}=r\left(v_{p}-D\right)$ is correlation coefficient, 0.858 , of $v_{p}-D$ relation; $r_{13}=r\left(v_{p}-P\right)$ is correlation coefficient, 0.288 , of $v_{p}-P$ relation; and $r_{23}=r(P-D)$ is correlation coefficient, 0.134 , of $(P-D)$ relation in figure 3 . Therefore, applying (6), we have $r_{12,3}=0.863$ implying that the observed $v_{p}-D$ correlation could not have arisen from a possible $v_{p}-P$ correlation.

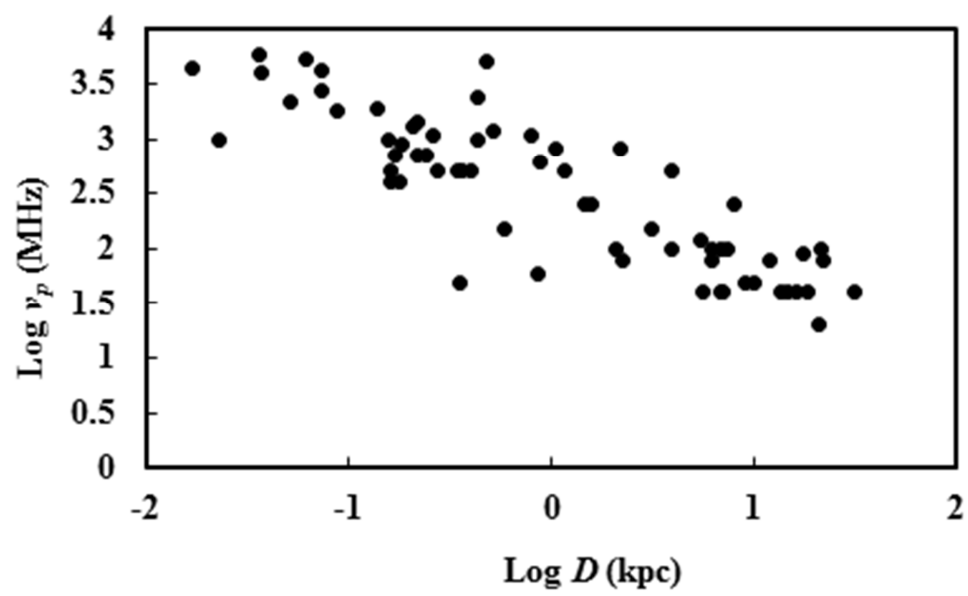

Figure 1. The scatter plot of spectral turnover against linear size.

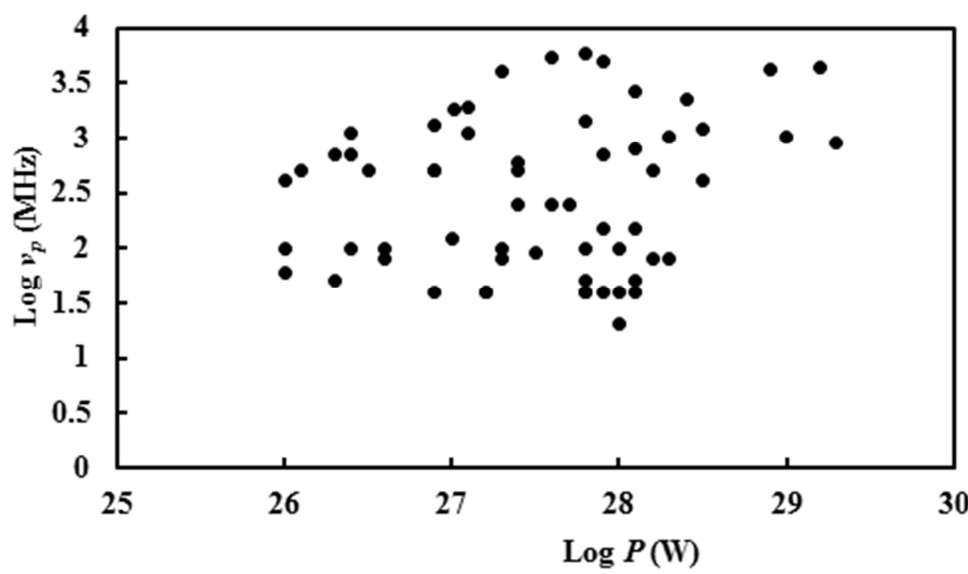

Figure 2. The scatter plot of spectral turnover against luminosity.

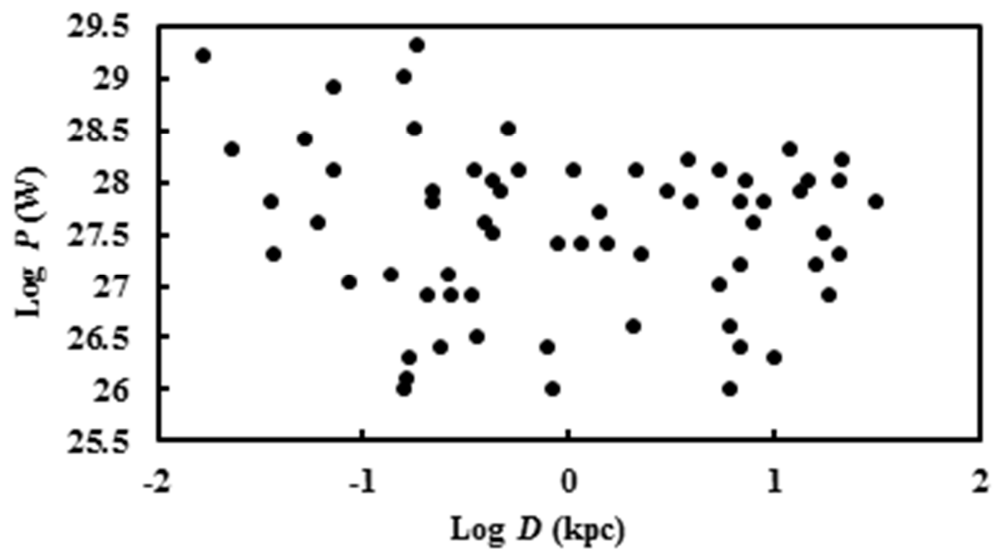

Figure 3. Scatter plot of Luminosity against linear size. 


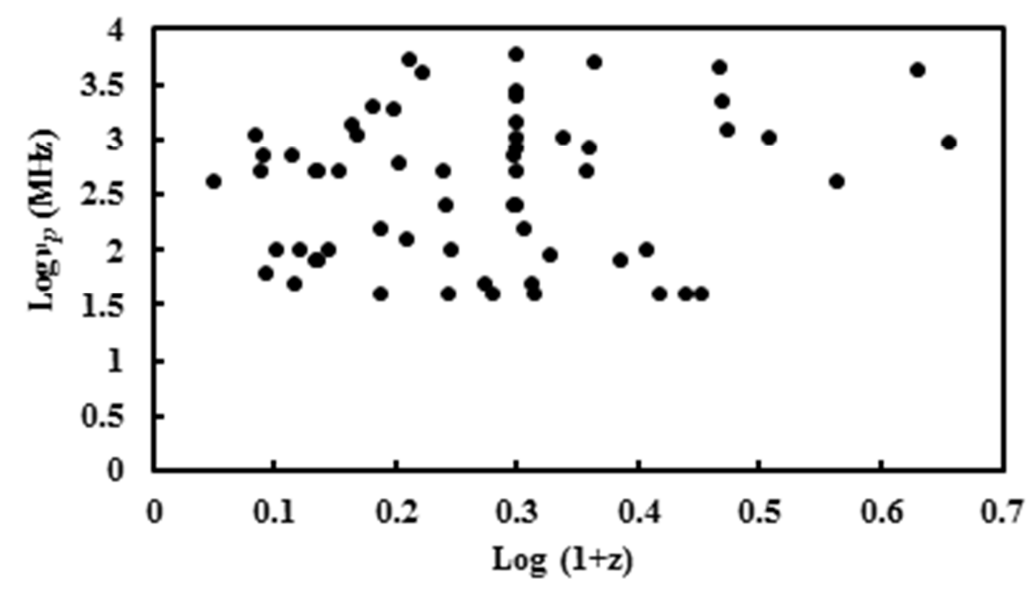

Figure 4. Scatter plot of spectral turnover against redshift.

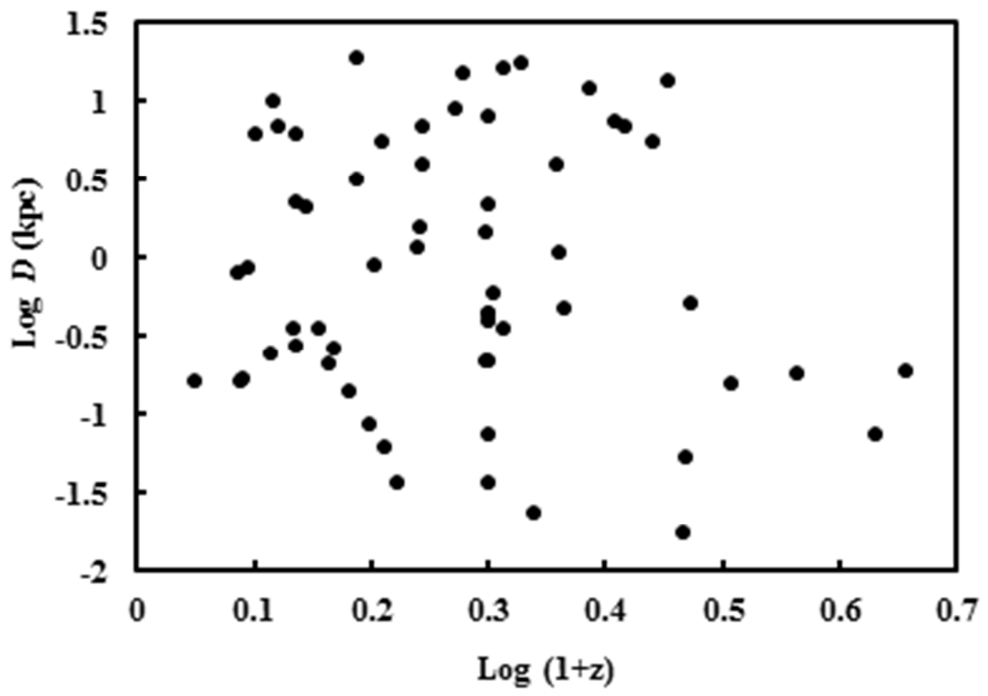

Figure 5. Scatter plot of linear size against redshift.

\section{Discussion}

The discovery of a strong correlation between the frequency peak, $v_{p}$, and linear sizes of Compact Steep Spectrum sources offers a very important diagnostics for the nature of the surrounding medium through which these sources are evolving. [30] has developed an analytical model for the evolution of CSSs through an ambient medium of density, $n_{e}$, and found that $v_{p}$ constitutes a characteristic signature of dense gases around these sources. This important result may however be an artifact if the peak frequency or linear size depends on other factors such as redshift, $z$, and/or radio luminosity, $P$, (in flux density limited samples, both $Z$ and $P$ are strongly correlated due to Malmquist bias).

Our results show only a marginally significant correlation between $v_{p}$ and $P$ (with correlation coefficient, $r \approx 0.3$ ) and no $D-z / P$ or $v_{p}-z$ correlation (see figures $3-5$ ). The observed marginal $v_{p}-P$ correlation cannot however account for the $v_{p}-D$ correlation. Furthermore, the absence of any $P-D$ correlation suggests that the source growth is independent of the luminosity. In more extended sources, the source size increases with radio power up to some maximum, and then decreases thereafter [27]. The implication of the present result is that the ambient medium through which the CSS sources propagate may be different from that of their more extended counterparts.

\section{Conclusion}

Among all the currently observable physical properties of Compact Steep Spectrum sources (CSSs), the frequency peak/linear size $\left(v_{p}-D\right)$ relation gives the strongest correlation with correlation coefficient, $r \approx-0.9$. This strong correlation suggests that the mechanism for the spectral turnover (frequency peak), $v_{p}$, lies in the source size, $D$. Hence, any good model for the evolution of CSSs should be able to explain $v_{p}-D$ strong correlation. However, in this present paper, we have shown that $v_{p}-D$ relation is not an artifact arising from either $v_{p}-P / Z$ or $D-P / z$ relations. The absence of $P-D$ correlation suggests that source growth is independent of the observed luminosity, implying that the mechanism for the spectral turnover may lie in the source size. 


\section{References}

[1] J. C. Cavalho, "The evolution of GHz-peaked spectrum radio sources", Astron. And Astrophy., vol. 329, pp. 845 - 852, 1998

[2] D. J. Saikia, S. Jeyakumar, P. J. Wiita, H. S. Sanghera, R. E. Spencer, "Compact steep spectrum radio sources and unification schemes”, Mont. Not. Roy. Ast. Rev., vol. 276, pp. $1215-1223,1995$

[3] F. Mantovani, A. Rossetti, W. Junor, D. J. Saikia, C. J. Salter, "Radio polarimetry of compact steep spectrum sources at sub-arcsecond resolution", Astron. and Astrophy., vol. 555, A4, pp. $1-12,2013$

[4] I. A. G. Snellen, R. T. Schillizzi, A. G. de Bruyn, G. K. Miley, "Deceleration of relativistic radio components and the morphologies of gigahertz peaked spectrum sources",Astron. And Astrophy., vol. 333, pp. 70 - 78, 1998a

[5] I. A. G. Snellen, R. T. Schillizzi, A. G de Bruyn, G. K. Miley, R. B. Rengelink, H. J. Röttgering, M. N. Bremer, "A new sample of faint gigahertz peaked spectrum radio sources", Astron. and Astrophy.,vol. 131, pp. 435 - 449, 1998b

[6] I. A. G. Snellen, R. T. Schillizzi, G. K. Miley, M. N. Bremer, H. J. Röttgering, H. J. van Langevelde, "Faint Gigahertz peaked spectrum sources and the evolution of young radio sources", New Astron. Rev. vol. 43, pp. 675 - 679, 1999

[7] C. Fanti, R. Fanti, D. C. Dallacasa, R. T. Schilizzi, R. E.Spencer, C. Stanghellini, "Are compact steep spectrum sources young?”Astron. and Astrophy., vol. 302, pp. 317 - 326, 1995

[8] F. Mantovani, W. Junor, R. Fanti, L. Padrielli, D. J. Saikia, "VLA polarimetry of compact steep spectrum sources", Astron. and Astrophy., vol. 125, pp 573 - 580, 1997

[9] C. P. O'Dea, “The compact steep spectrum and Gigahertz peaked spectrum radio sources", Pub. Astron. Soc. Pacific.Vol. 110,pp. $493-532,1998$

[10] A. C. S. Readhead, "Evolution of Powerful Extragalactic Radio Sources", Proc. National Academy of Sciences, pp 11447 11450, 1995

[11] I. Owsianik, J. E. Conway, "First detection of hotspot advance in a compact symmetric object: Evidence for a class of very young extragalactic radio sources", Astron. and Astrophy., vol. 337, pp. $69-79,1998$

[12] M. Murgia, R. Fanti, L. Gregorini, U. Klein, K. H. Mark, M. Vigotti, "Synchrotron spectra and ages of compact steep spectrum radio radio sources", Astron. and Astrophy. vol. 345, pp. $769-777,1999$

[13] A. Polatidis, P. N. Wilkinson, W. Xu, A. C. S. Readhead, T. J. Pearson, G. B. Taylor, R. C. Vermeulen, "Compact symmetric objects in a complete flux density limited sample" N.A.R. 43, pp $657-661,1999$

[14] S. Tinti, G. De Zotti, "Constraints on evolutionary properties of $\mathrm{GHz}$ peaked spectrum galaxies", Astron. and Astrophy. vol. 445, pp. $889-899,2006$

[15] A. Marecki, A. Sokołowska, "Multi-epoch VLBA observations of radio galaxy 0932+075: is this a compact symmetric object?", Astron. and Astrophy., vol. 569, A22, pp. 1 - 6, 2014
[16] G. Stanghellini, D. Dallacasa, C. P. O’Dea, S. A. Baum, R. Fanti, C. Fanti, "VLBA observations of GHz-peaked spectrum radio sources at 15GHz", Astron. and astrophy. vol. 377, pp. $377-388,2001$

[17] D. Dallacasa, S. Tinti, C. Fanti, L. Fanti, L. Gregorini, C. Stanghellini, M. Vigotti, "The B3-VLA CSS sample II. VLBA images at 18cm”, Astron. and astrophy., vol. 389, pp. $115-125$, 2002

[18] P. Augusto, J. I. Gonzalez-Serrano, A. C. Edge,N. A. B. Gizani, P. N. Wilkinson, I. Perez-Fouron, "The Kpc-scale source population”, New Astron. Rev., vol. 43, pp. 663 - 667, 1999

[19] A. Labiano, "Tracing jet-ISM interaction in young AGN: correlations between [O III] $\lambda 5007 \dot{A}$ and $5 \mathrm{GHz}$ emission", Astron. and Astrophy., vol. 488, pp. L59 - L62, 2008

[20] G. Stanghellini, C. P. O’Dea, D. Dallacasa, S. A. Baum, R. Fanti, C. Fanti, "A complete sample of GHz-Peaked spectrum radio sources and its radio properties", Astron. and Astrophy. vol. 131 , pp. $303-315,1998$

[21] A. C. S. Readhead, G. B. Taylor, W. Xu, T. J. Pearson, P. N. Wilkinson, A. G. Polatidis, "The statistics and ages of compact symmetric objects" Astrophy. Journal, vol. 460, pp. 612 - 633, 1996

[22] N. Kawakatu, M. Kino, "The velocity of large-scale jets in a declining density medium". RevMex AA(Serie de Conferencias), vol. 27, pp. $192-197,2007$

[23] C. Stanghellini, C. P. O'Dea, D. Dallacasa, P. Cassaro, S. A. Baum, R. Fanti, C. Fanti, C., "Extended emission around GPS radio sources", Astron. and Astrophy., vol. 443, pp. 891 - 902, 2005

[24] A. Labiano, C. P. O’Dea, R. Gelderman, W. H. de Vries, D. J. Axon, P. D. Barthel, S. A. Baum, A. Capetti, R. Fanti, A. M. Koekemoer, R. Morganti, C. N. Tadhunter, "HST/STIs low dispersion spectroscopy of three compact steep spectrum sources", Astron. and Astrophy., vol. 436, pp. 493 - 501, 2005

[25] S. Jeyakumar, P. J. Wiita, D. J. Saikia, J. S. Hooda, "Jet propagation and the asymmetries of CSS radio sources", Astron. and Astrophy. vol. 432, pp. 823 - 833, 2005

[26] S. E. Okoye, L. I. Onuora, "Angular diameter-redshift relation for extragalactic radio sources", Astrophy. Journal, vol. 260, pp. $37-43,1982$

[27] A. A. Ubachukwu, J. N.Ogwo, "Luminosity selection effects and linear size evolution in the quasar/galaxy unification scheme", Aust. Journ. of Phy., vol. 51, pp. 143 - 151, 1998

[28] A. K. Singal, "Evidence against the unified scheme for powerful radio galaxies and quasars", Mont. Not. Roy. Astron. Soc., vol. 262, pp. L27 - L30, 1993

[29] R. Nilson, M. J. Valtonen, T. Jaakola, "On the redshift-apparent size diagram of double radio sources", Astrophy. Journ., vol. 413 , pp. $453-476,1993$

[30] J. C. Ezeugo, A. A. Ubachukwu, "The spectral turnover-linear size relation and the dynamical evolution of compact steep spectrum sources", Mont. Not. Roy. Astron. Soc., vol. 408, pp. $2256-2260,2010$ 